\title{
Resolving sorting mechanisms into exosomes
}

\author{
Cell Research (2015) 25:531-532. doi:10.1038/cr.2015.39; published online 31 March 2015
}

The complexity of mechanisms driving protein sorting into exosomes is only beginning to emerge. In a paper recently published in Cell Research, Roucourt et al. report that trimming of heparan sulfate side chains of syndecans by endosomal heparanase facilitates sorting into exosomes by the formation of tight syndecan clusters that are recruited by the multivalent adaptor syntenin to the ALIX-ESCRT sorting machinery at endosomes.

Multicellular organisms function by virtue of intercellular communication, which can be accomplished through direct cell-cell contact or transfer of secreted molecules. A third mode of communication, which is increasingly recognized, involves extracellular vesicles (EVs) [1]. EVs are composed of integrated molecular packages of membrane proteins, cytosolic proteins, lipids, and RNA, and can transmit complex messages from donor to specific target cells. EVs have been implicated in many different physiological processes, including immune regulation, stem cell regulation, tissue morphogenesis, and gamete function, but also in disease such as cancer progression and metastasis, and neurodegeneration. EVs can be released from donor cells by shedding from the plasma membrane, and these are then generally referred to as microvesicles (MVs). In contrast, EVs that are secreted by multivesicular endosomes (MVEs) are called exosomes. The intraluminal vesicles of MVEs are generated by inward budding of the endosomal limiting membrane (Figure 1 ), and hence exosomes have the same topology as MVs and cells, with their exoplasmic side exposed.

Although physiological functions of EVs have nowadays been proposed in $>9000$ reports, MVs and exosomes are usually not discerned. This is mainly due to lack of knowledge on molecular mechanisms that drive or regulate the sorting of molecules into MVs or exosomes, and this also hampers the design of experiments to demonstrate in vivo relevance of EVs in intercellular communication. Molecular mechanisms for exosome formation at endosomes involve the endosomal-sorting complex required for transport (ESCRT) machinery. ESCRT was originally identified to drive the sorting of ubiquitin-conjugated membrane proteins into vesicles that bud into the lumen of a distinct set of MVEs that ultimately fuse with lysosomes rather than with the plasma membrane, resulting in the degradation of their vesicular contents. ESCRT is composed of 4 multiprotein sub-complexes, of which ESCRT-0, -I and -II recognize and sequester ubiquitinated membrane proteins at the endosomal delimiting membrane, while ESCRT-III drives membrane budding and actual scission of intraluminal vesicles [2]. Sorting of proteins into exosomes, however, appears to occur independently of cargo ubiquitination, and only a selected number of ESCRT components are involved in exosome formation [3]. For example, ubiquitination of MHC class II is required for its sorting into intraluminal vesicles of lysosome-targeted MVEs, but not for incorporation into exosomes [4]. Sorting of the transferrin receptor to exosomes, which occurs efficiently only in reticulocytes, is also independent of ubiquitination, but instead relies on direct binding of its cytoplasmic domain to the ESCRT accessory protein ALIX [5].

The research group of Guido David has previously shown that sorting of membrane proteins of the syndecan family into exosomes also involves ALIX, but in that case is linked to ALIX via the cytosolic adaptor syntenin [6]. Syntenin couples to membrane proteins with two PDZ domains and to ALIX with three LYPX $_{\mathrm{n}} \mathrm{L}$ motifs, and ALIX binds on its turn to ESCRT-III, the machinery responsible for intraluminal vesicle formation at MVEs. Syndecans form complexes through lateral interactions between their attached heparan sulfate polysaccharide chains. Guido David and co-workers have now shown that heparanase activity in endosomes trims long heparan sulphate chains into shorter ones, allowing clustering of syndecans. Further condensation is achieved as a consequence of removal of the syndecan luminal domain by endosomal proteases, leaving the membrane-embedded C-terminal domain. Heparanase-induced clustering is thought to stimulate the binding of syndecan cytoplasmic domains to the tandem PDZ domains of syntenin, driving ALIX-ESCRT-mediated sorting into exosomes. Interestingly, heparanase activity also facilitated the recruitment of CD63 into exosomes, in a syntenindependent manner [6,7]. Sorting of many membrane proteins into exosomes coincides with their association with tetraspanin membrane proteins [8]. Webs of interacting tetraspanins and associated proteins are stabilized by protein palmitoylation, and lipids also play an important role in the forma- 

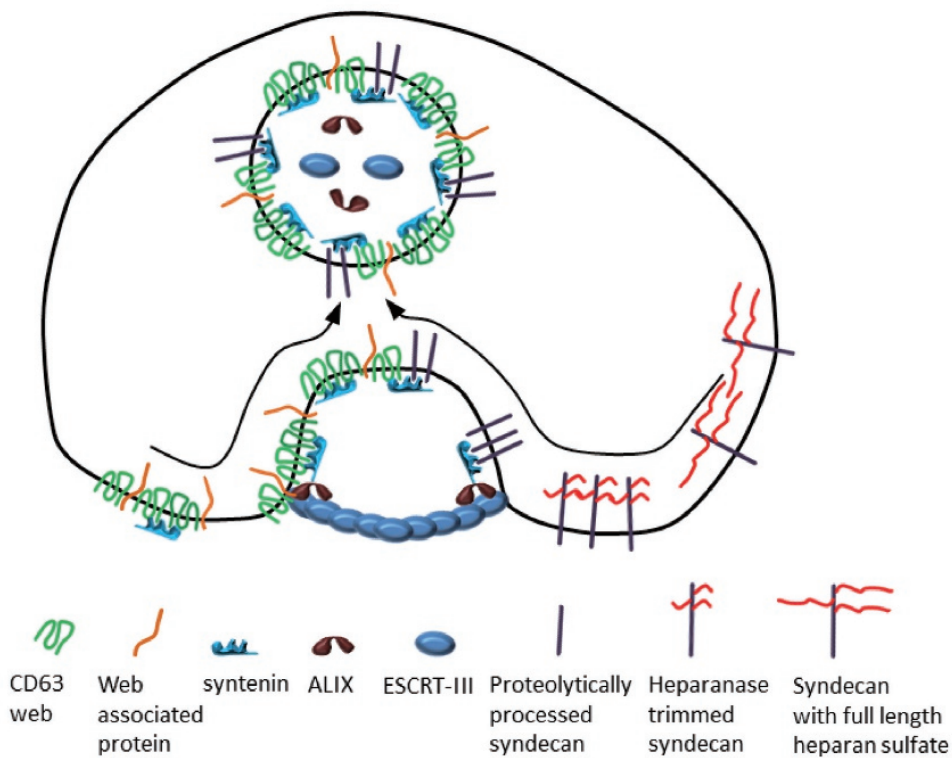

Figure 1 Syndecans are processed at endosomes, first by trimming associated heparin sulfate site chains, followed by proteolytic cleavage. Clustered syndecan can now be recruited by multivalent syntenin, which on its turn is coupled to the ESCRT machinery via ALIX. Similarly, CD63 is recruited by syntenin, and with it presumably other membrane proteins that are associated with tetraspanin webs. Heparanase also stimulates sorting of CD63, indicating that the two pathways driven by syndecan and CD63 are somehow integrated.

tion of tetraspanin webs, conceivably explaining the relative enrichment of cholesterol and glycosylceramides in exosomes, as well as the dependency on sphingomyelinase activity for exosome formation [9]. Non-tetraspanin membrane proteins may in this way piggyback onto tetraspanin webs for their sorting into exosomes [8]. Interestingly, the tetraspanin CD63, which is highly enriched in exosomes and considered to be important for chaperoning cargo into exosomes [10], can also be recruited by syntenin [11]. Sorting of tetraspanin webs at endosomes into exosomes could thus, similar to syndecans, be driven by the cytoplasmic adaptor syntenin, and the recruitment by syntenin of tetraspanin webs and syndecan clusters are thus integrated processes (Figure 1).

All in all, a complex picture is emerging, in which both CD63 and syndecans, and possibly other membrane proteins that associate with endosomal syndecan and/or tetraspanin-enriched microdomains, are sorted into exosomes by a shared syntenin-ALIX-ESCRT machinery.

Importantly, not all EV cargoes appear to depend on heparanasesyntenin-ALIX-ESCRT, as exemplified by flotillin, CD9 and CD81 [6, 7]. One possible explanation is that distinct but parallel sorting mechanisms exist that drive cargo into a single population of exosomes. Another possibility is that separate sorting mechanisms drive the formation of distinct $\mathrm{EV}$ populations containing different cargo molecules. For example, syntenin-dependent and -independent EVs may be represented by exosomes and plasma membranederived MVs, respectively. The origin of EVs, exosomes or MVs, is ill defined in most research. The current study by Roucourt and coworkers [7] provides molecular tools, supplementation with heparanase to stimulate, or syntenin depletion to interfere with, cargo incorporation into exosomes, that could help researchers to establish the precise origin of EVs, as well as their roles in biological processes.

\section{Willem Stoorvoge ${ }^{1}$}

${ }^{1}$ Utrecht University, Faculty of Veterinary Medicine, Dept. of Biochemistry \& Cell Biology, Yalelaan 2, 3584 CM, The Netherlands E-mail: W.Stoorvoge1@uu.nl

\section{References}

1 Raposo G, Stoorvogel W. J Cell Biol 2013; 200:373-383.

2 Raiborg C, Stenmark H. Nature 2009; 458:445-452.

3 Colombo M, Moita C, van Niel G, et al. J Cell Sci 2013; 126(Pt 24):5553-5565.

4 Buschow SI, Nolte-'t Hoen EN, van Niel G, et al. Traffic 2009; 10:1528-1542.

5 Géminard C, De Gassart A, Blanc L, et al. Traffic 2004; 5:181-193.

6 Baietti MF, Zhang Z, Mortier E, et al. Nat Cell Biol 2012; 14:677-685.

7 Roucourt B, Meeussen S, Bao J, et al. Cell Res 2015; 25:412-428.

8 Andreu Z, Yáñez-Mó M. Front Immunol 2014; 5:442.

9 Trajkovic K, Hsu C, Chiantia S, et al. Science 2008; 319:1244-1247.

10 van Niel G, Charrin S, Simoes S, et al. Dev Cell 2011; 21:708-721.

11 Latysheva N, Muratov G, Rajesh S, et al. Mol Cell Biol 2006; 26:7707-7718. 